\title{
Biomarcadores y mortalidad por SARS-CoV-2 en pacientes del servicio de urgencias respiratorias
}

\author{
Petrona I. Sánchez-Sánchez ${ }^{1}$, Patricia Seefoo-Jarquin², Ma. de la Luz León-Vázquez ${ }^{2 *}$, \\ Erik Castillo-de la Peña ${ }^{2}$ y Dulce Cuatepotzo-Xochihua ${ }^{1}$ \\ ${ }^{1}$ Hospital General de Zona N. ${ }^{0}$; ${ }^{2}$ Hospital General de Subzona N. ${ }^{\circ}$ 8. Instituto Mexicano del Seguro Social, Tlaxcala, México
}

\begin{abstract}
Resumen
Introducción: Por la relación con la inflamación sistémica causada por la infección por el coronavirus 2 del síndrome respiratorio agudo grave (SARS-CoV-2), el recuento de linfocitos, neutrófilos y la relación neutrófilos/linfocitos se han propuesto como marcadores de severidad y mortalidad. Objetivo: Analizar la relación entre biomarcadores y la mortalidad en los pacientes con SARS-CoV-2 que acudieron al servicio de urgencias. Métodos: Diseño observacional, retrospectivo y analitico. Se examinaron expedientes de pacientes confirmados con SARS-CoV-2 que acudieron al servicio de urgencias. El recuento de plaquetas, leucocitos, hemoglobina, neutrófilos, linfocitos e índice neutrófilos/linfocitos se comparó entre los que fallecieron y sobrevivieron. Se utilizó el programa estadístico SPSS versión 24 para el análisis; se incluyó estadística descriptiva, $\chi^{2}, U$ de Mann-Whitney y prueba t para grupos independientes. Se consideró significativa una $p$ valor menor a 0.05 . Resultados: Se analizaron 231 expedientes, el 63\% de hombres. La edad de los pacientes fue entre 24 y 90 años. El 39\% requirieron apoyo ventilatorio mecánico. El 5.6\% cursó con lesión renal aguda. El 56\% fallecieron. El índice neutrófilos/linfocitos, leucocitosis y linfopenia se asociaron significativamente con la mortalidad $(p<0.05)$, no así el recuento plaquetario 0 la anemia. Conclusiones: La mortalidad en pacientes con SARS-CoV-2 está asociada con el índice neutrófilos/linfocitos, leucocitosis y linfopenia.
\end{abstract}

Palabras clave: Marcador de laboratorio. Biomarcadores. SARS-CoV-2.

\section{Mortality biomarkers of SARS-CoV-2 in patients in the respiratory emergency department}

\section{Abstract}

Background: Due to the relationship with the systemic inflammation caused in SARS-CoV-2, the count of lymphocytes, neutrophils and the neutrophillymphocyte ratio have been proposed as markers of severity and mortality. Objective: To analyze the relationship between biomarkers and mortality in patients with SARS-CoV-2 who attended the emergency department. Methods: Observational, retrospective, analytical design. Records of patients confirmed with SARS-CoV-2 who attended the emergency department were examined. Platelet, leukocyte, hemoglobin, neutrophil, lymphocyte count, and neutrophil/lymphocyte ratio were compared between those who died and survived. The SPSS version 24 statistical program was used for the analysis, it included descriptive statistics, chi square, Mann Whitney $U$ and $T$ for independent groups. A p value less than 0.05 was considered significant. Results: 231 files were analyzed, $63 \%$ male. The age of the patients was between 24 and 90 years. $39 \%$ required mechanical ventilatory support. 5.6\% had acute kidney injury. $56 \%$ died. The neutrophilllymphocyte

\section{Correspondencia:}

*Ma. de la Luz León-Vázquez

E-mail: clarissa023@gmail.com
Disponible en internet: 30-07-2021

Fecha de recepción: 24-03-2021

Fecha de aceptación: 07-05-2021 DOI: 10.24875/REIE.21000022
Rev Educ Investig Emer. 2021;3(2):52-58 www.medicinadeemergencias.com

列 bajo la licencia CC BY-NC-ND (http://creativecommons.org/licenses/by-nc-nd/4.0/). 
index, leukocytosis, and lymphopenia were significantly associated with mortality $(p<0.05)$, not the platelet count or anemia. Conclusions: Mortality in patients with SARS-CoV-2 is associated with the neutrophillymphocyte index, leukocytosis and lymphopenia.

Key words: Laboratory Marker. Biomarkers. SARS-CoV-2.

\section{Introducción}

La infección por coronavirus 2 del síndrome respiratorio agudo grave (SARS-CoV-2) puede presentarse clínicamente de leve a grave, caracterizada por síndrome de dificultad respiratoria aguda y falla orgánica multisistémica, la mayoría de las veces con desenlaces fatales ${ }^{1}$.

Por ser una enfermedad emergente, sus características aún siguen en estudio, su historia natural y cambios fisiopatológicos, sobre todo inmunológicos, pueden ayudar a tomar decisiones en el manejo, así como identificar a las personas con mayor riesgo?2.

Es importante optimizar la atención de los pacientes, por lo que es necesario identificar marcadores séricos, clínicos y paraclínicos que sirvan como pronósticos para estratificar el riesgo de mortalidad. Entre los parámetros hematológicos propuestos por su relación con la inflamación sistémica están el recuento de linfocitos, neutrófilos y el índice neutrófilos/linfocitos $(\mathrm{INL})^{3}$.

Un recuento linfocitario menor a 1,000 se ha asociado con enfermedad grave, lo que sugiere que el SARSCoV-2 podría actuar principalmente en los linfocitos $T$, induciendo una tormenta de citocinas, inhibiendo la función inmunitaria celular del cuerpo ${ }^{4,5}$. Como factores de riesgo para muerte en pacientes con neumonía por SARS-CoV-2 se han identificado la edad mayor a 65 años, enfermedades cardiovasculares o cerebrovasculares preexistentes y linfocitosis 6 .

Existen algunas controversias en relación con otros marcadores propuestos, como son los niveles de hemoglobina $(\mathrm{Hb})$ y plaquetas ${ }^{7}$. Por ello el objetivo del estudio fue analizar si existe correlación entre biomarcadores y la mortalidad de los pacientes con SARSCoV-2 que acudieron al servicio de urgencias respiratorias del Hospital General de Zona N. ${ }^{\circ} 1$ Tlaxcala.

\section{Pacientes y métodos}

Se utilizó un diseño observacional, analítico, transversal y retrospectivo. Previa autorización por el personal directivo y el comité de ética en investigación y comité local de investigación en salud con registro
R-200-2902-019, se analizaron expedientes clínicos de los pacientes que acudieron al servicio de urgencias respiratorias adultos con diagnóstico de enfermedad por coronavirus 2019 (COVID-19) en el Hospital General de Zona N. ${ }^{\circ} 1$ de Tlaxcala, México. Por el diseño de estudio y ausencia de intervención no se solicitó consentimiento informado.

- Criterios de inclusión de pacientes: se incluyeron expedientes de pacientes mayores de 18 años, sexo indistinto con signos y síntomas de infección respiratoria baja, hallazgos radiológicos compatibles de infección viral y prueba positiva para SARS-CoV-2, que acudieron al servicio de urgencias entre marzo y octubre del 2020. Se excluyeron aquellos que fueron trasladados a otra unidad hospitalaria o solicitaron alta voluntaria.

- Recopilación de datos: se extrajeron datos generales tales como edad, sexo, ingreso y egreso hospitalario, signos vitales (frecuencia cardiaca, respiratoria, presión arterial), escala de Glasgow reportada y resultados de laboratorio $(\mathrm{Hb}$, leucocitos, neutrófilos, linfocitos, plaquetas), así como saturación de oxígeno $\mathrm{PaO}_{2} / \mathrm{FIO}_{2}$.

- Medidas de resultado: la severidad de la infección por SARS-CoV-2 se evaluó con la puntuación SOFA (Sequential Organ Failure Assessment). El egreso hospitalario se dividió en alta por mejora o defunción. Los biomarcadores de laboratorio considerados fueron $\mathrm{Hb}$, leucocitos, neutrófilos, linfocitos, INL y plaquetas.

- Análisis estadístico: las variables continuas (edad, $\mathrm{Hb}$, leucocitos, neutrófilos, linfocitos, plaquetas) se presentan con media y desviación estándar $( \pm)$, las variables categóricas (sexo, egreso hospitalario, puntuación SOFA) se presentan con frecuencias y porcentajes. La distribución de los datos se valoró mediante la prueba de Kolmogorov-Smirnov utilizando pruebas no paramétricas ( $U$ de Mann-Whitney) y se empleó la prueba t para muestras independientes para relacionar los biomarcadores $(\mathrm{Hb}$, leucocitos, neutrófilos, linfocitos, plaquetas) con el egreso hospitalario. Se calculó la odds ratio (OR) para mortalidad en relación con los factores que mostraron diferencia significativa. Se realizaron curvas ROC (receiver operating characteristic) para determinar el 


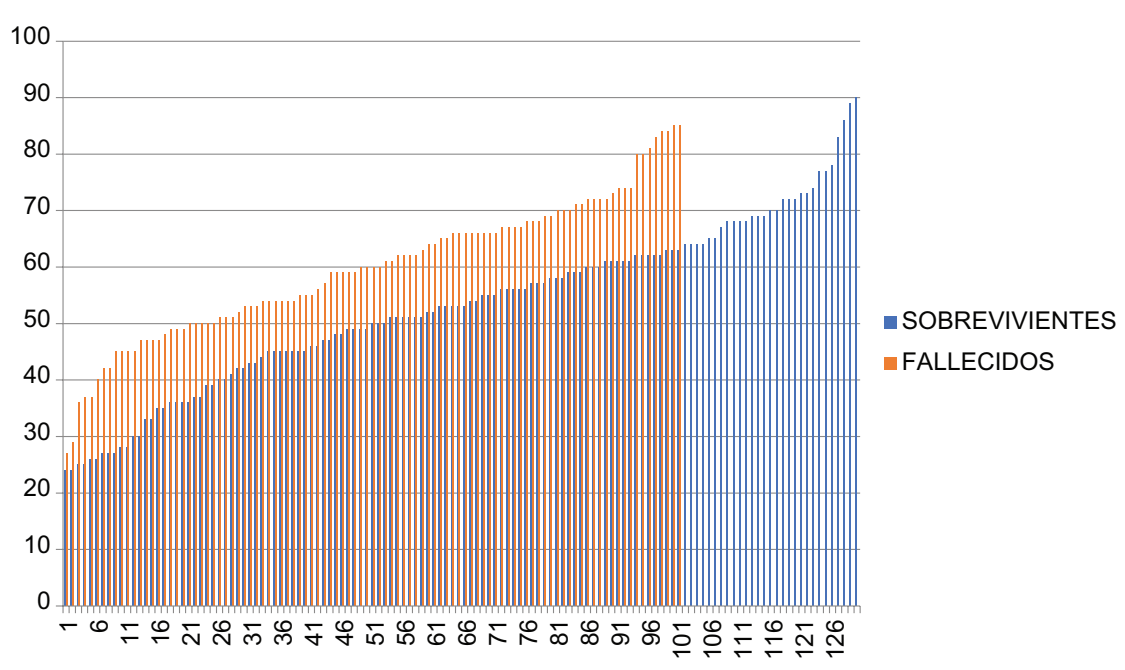

Figura 1. Relación entre edad y mortalidad.

punto de corte para el INL, tanto para defunción como para necesidad de apoyo ventilatorio mecánico (AVM). Un valor de $p$ menor a 0.05 fue considerado significativo. Se utilizó el programa estadístico SPSS versión 24 para el análisis.

\section{Resultados}

Se analizaron un total de 268 expedientes, se excluyeron 37 por no contar con datos completos de laboratorio. Ochenta y cinco (37\%) correspondieron a expedientes de mujeres y 146 (63\%) de hombres. La edad de los pacientes fue entre 24 y 90 años, con media de $56 \pm 14$ años, con distribución no normal (Kolmogorov-Smirnov 0.000).

La estancia hospitalaria fue entre cero y 44 días, con una media de $9 \pm 7$ días, mediana de siete días y rango intercuartil (IQR) 4 -12.

Ochenta y siete casos (32\%) tenían antecedente de diabetes mellitus tipo 2 y/o hipertensión arterial sistémica, $118(51 \%)$ no presentaban comorbilidades.

La saturación de oxígeno al ingreso fue entre 34 y 99\%, mediana 90\% (IQR: 85-94). Noventa (39\%) pacientes requirieron $\mathrm{AVM}, 13(5.6 \%)$ con lesión renal aguda concomitante, siete de ellos fueron manejados con hemodiálisis de rescate y seis con diálisis peritoneal. Fallecieron 130 pacientes (56\%).

El sexo, AVM y comorbilidades se asociaron significativamente con la defunción $\left(\chi^{2} p=0.049,0.000\right.$ y 0.005 respectivamente), el sexo masculino no se consideró factor de riesgo para mortalidad (OR: 1.378; intervalo de confianza del 95\% [IC 95\%]: 0.988-1.922).
Tabla 1. Pronóstico de mortalidad con base en puntuación SOFA (Sequential Organ Failure Assessment)

\begin{tabular}{|c|c|c|c|}
\hline Puntuación & \multicolumn{2}{|c|}{ Porcentaje de mortalidad } & n (\%) \\
\hline $0-6$ & \multicolumn{2}{|c|}{$<10 \%$} & $185(80)$ \\
\hline 7-9 & \multicolumn{2}{|c|}{$15-20 \%$} & $16(8)$ \\
\hline 10-12 & \multicolumn{2}{|c|}{$40-50 \%$} & $9(4)$ \\
\hline $13-14$ & \multicolumn{2}{|c|}{$50-60 \%$} & $12(5)$ \\
\hline 15 & \multicolumn{2}{|c|}{$>80 \%$} & $4(2)$ \\
\hline $16-24$ & \multicolumn{2}{|c|}{$>90 \%$} & $2(1)$ \\
\hline & Fallecieron & Sobrevivieron & $\chi^{2}$ \\
\hline 0 a 2 & $19(8)$ & $47(20)$ & 0.004 \\
\hline 3 o más & $82(35)$ & $83(36)$ & \\
\hline
\end{tabular}

Al comparar la mediana de edad entre los grupos (fallecidos vs. sobreviviente) se observó diferencia significativa (U de Mann-Whitney: 4,776; $p=0.000$ ) (Fig. 1).

La gravedad de los pacientes se evaluó mediante la puntuación SOFA, que estuvo entre 0 y 16, mediana 4. Ciento ochenta y cinco $(80 \%)$ tenían una probabilidad de muerte menor al $10 \%$, al comparar la mortalidad con la puntuación SOFA, con punto de corte en dos, se observa diferencia significativa (Tabla 1).

Los resultados de la citometría hemática se muestran en la tabla 2.

Se compararon las medias de los valores de la citometría hemática entre los pacientes que fallecieron y 
Tabla 2. Hallazgos de laboratorio. Citometría hemática*

\begin{tabular}{l|c} 
Hemoglobina g/dl & $14 \pm 2$ \\
Recuento de glóbulos blancos x $10^{9} / /$ & $12,285 \pm 7,473$ \\
Recuento de neutrófilos $10^{9} / /$ & $10,411 \pm 7,065$ \\
Recuento de linfocitos $10^{9} / /$ & $1,255 \pm 1,149$ \\
Recuento de plaquetas $10^{9} / l$ & $257,961 \pm 113,163$ \\
Índice neutrófilos/linfocitos & $12 \pm 10$
\end{tabular}

*Media \pm gramos/decilitro.

sobrevivieron, observando diferencia significativa en relación con el INL (Tabla 3). Con base en los resultados de la citometría hemática, considerando los valores de referencia del laboratorio y sexo se clasificó en forma dicotómica la presencia de anemia $(\mathrm{Hb}<12 \mathrm{~g} / \mathrm{dL}$ para mujeres y $\mathrm{Hb}<13 \mathrm{~g} / \mathrm{dL}$ para hombres), leucocitosis, trombocitopenia y linfopenia, observando diferencia significativa en relación con la leucocitosis y linfopenia, siendo factores de riesgo para defunción. Ningún paciente presentó niveles menores a 1,500 de neutrófilos (Tabla 4).

El área bajo la curva en la curva ROC para el INL fue de 0.615 con IC 95\%: 0.541-0.689 para defunción (Fig. 2) y de 0.590 con un IC 95\%: 0.513-0.667 para el requerimiento de AVM (Fig. 3). Considerando un punto de corte de 4.85, con una sensibilidad de 87 y especificidad de 76 , se observa diferencia significativa en relación con la mortalidad $\left(\chi^{2} p=0.035\right)$, no así con la necesidad de AVM $(p=0.280)$.

\section{Discusión}

En el contexto de la pandemia por SARS-CoV-2 se ha tratado de predecir la gravedad y mortalidad de la enfermedad por medio de diversos marcadores. Aún con ciertas controversias en relación con los marcadores bioquímicos, no solo a la presencia de sus alteraciones, sino también a los puntos de corte.

La citometría hemática es una prueba accesible, la cual se solicita en forma casi sistemática en los servicios de urgencias. Nuestros hallazgos sugieren que la leucocitosis, la linfopenia y el aumento del INL pueden ser utilizados como predictores de mortalidad en pacientes con COVID-19.

En pacientes con infección por SARS-CoV-2 se espera la presencia de leucocitosis y linfopenia por la respuesta inflamatoria que conlleva. En un metaanálisis, Soraya y Ulhaq ${ }^{8}$ reportan una relación significativa entre pacientes con COVID-19 severa y la presencia de leucocitosis, linfopenia y trombocitopenia, así como

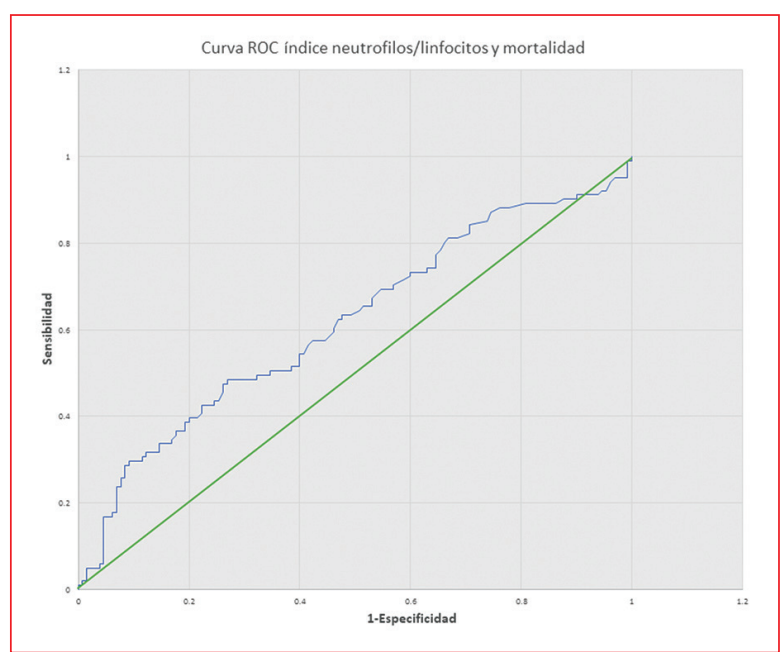

Figura 2. Índice neutrófilos/linfocitos y mortalidad.

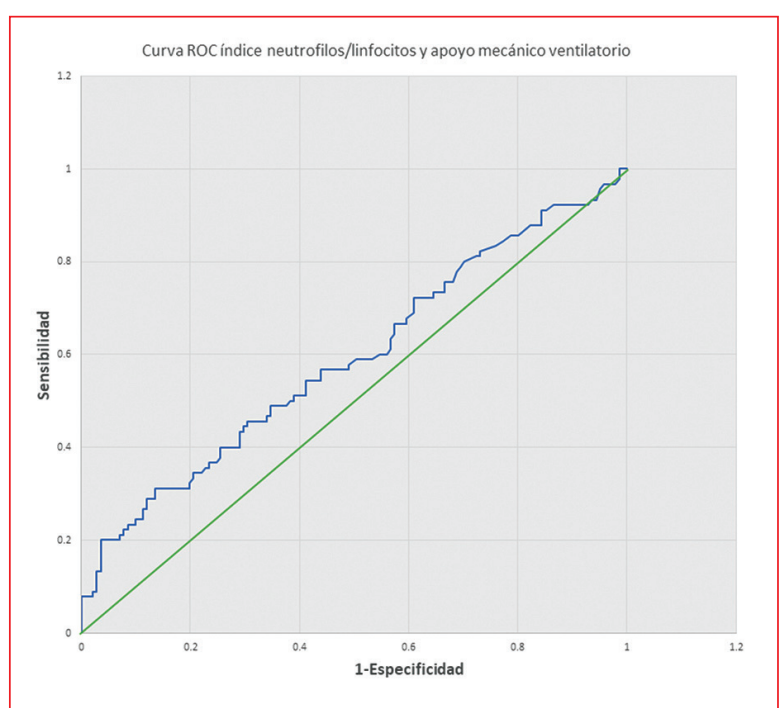

Figura 3. Índice neutrófilos/linfocitos y requerimiento de apoyo ventilatorio mecánico.

elevación neutrófilos, dímero $D$ y proteína $C$ reactiva (PCR). Coincidiendo con los hallazgos en los resultados respecto a la leucocitosis y linfopenia, no así con la trombocitopenia ni la neutrofilia. Esto puede deberse a la naturaleza de los estudios, el tipo de población y objetivo, que en este caso era diferenciar entre la mortalidad y en el metaanálisis entre la severidad de la enfermedad.

Ghahramani, et al. ${ }^{9}$, en otro metaanálisis en población asiática, muestran una significativa disminución de linfocitos, $\mathrm{Hb}$, plaquetas y aumento en el INL en 
Tabla 3. Diferencia de medias de citometría hemática en relación con el motivo de egreso

\begin{tabular}{|c|c|c|c|c|c|}
\hline & \multicolumn{3}{|c|}{ Motivo de egreso } & \multicolumn{2}{|c|}{$95 \%$ de intervalo de confianza de la diferencia } \\
\hline & $\begin{array}{l}\text { Defunción } \\
130(56 \%)\end{array}$ & $\begin{array}{c}\text { Alta } \\
101(44 \%)\end{array}$ & $\mathbf{p}^{*}$ & Inferior & Superior \\
\hline & \multicolumn{5}{|c|}{ Media \pm DE } \\
\hline Hemoglobina & $13.6 \pm 2.7$ & $13.9 \pm 2.4$ & 0.523 & -0.8908 & 0.4539 \\
\hline Leucocitos & $13,071 \pm 7,472$ & $11,675 \pm 7,445$ & 0.159 & -552.9 & 3,345 \\
\hline Neutrófilos & $11,017 \pm 6,704$ & $9,940 \pm 7,324$ & 0.251 & -768 & 2,922 \\
\hline Linfocitos & $1,264 \pm 1,309$ & $1,248 \pm 1,012$ & 0.917 & -294.7 & 327.4 \\
\hline Plaquetas & $256,285 \pm 122,571$ & $259,264 \pm 105,745$ & 0.843 & $-32,615.78$ & $26,658.65$ \\
\hline Índice neutrófilos /linfocitos & $14.3 \pm 11.4$ & $10.3 \pm 8.6$ & 0.004 & 1.34554 & 6.749 \\
\hline
\end{tabular}

*Prueba t para muestras independientes.

DE: desviación estándar.

Tabla 4. Factor de riesgo en relación con la mortalidad por SARS-CoV-2

\begin{tabular}{|l|l|c|c|c|c|}
\hline & & $\begin{array}{c}\text { Defunción } \\
\mathbf{1 3 0}(\mathbf{5 6} \%)\end{array}$ & $\begin{array}{c}\text { Sobrevivieron } \\
\mathbf{1 0 1}(\mathbf{4 4 \% )}\end{array}$ & $\mathbf{p}^{*}$ & OR (IC 95\%) \\
\hline Anemia & Con & $33(14 \%)$ & $34(15 \%)$ & 0.279 & $0.842(0.621-1.140)$ \\
\hline & Sin & $68(29 \%)$ & $96(42 \%)$ & & $1.445(1.064-1.963)$ \\
\hline Leucocitosis & Con & $62(27 \%)$ & $59(25 \%)$ & $\mathbf{0 . 0 1 6}$ & $1.521(1.111-2.082)$ \\
\hline Linfopenia & Sin & $39(17 \%)$ & $71(31 \%)$ & & $\mathbf{0 . 0 2 2}$ \\
\hline Trombocitopenia & Con & $21(9 \%)$ & $13(5 \%)$ & & $1.153(0.796-1.670)$ \\
\hline
\end{tabular}

*Prueba $\chi^{2}$ de Pearson.

OR: odds ratio; IC: intervalo de confianza; SARS-CoV-2: coronavirus 2 del síndrome respiratorio agudo grave.

pacientes con COVID-19 severa, sin demostrar diferencia en la leucocitosis. $\mathrm{Li}$, et $\mathrm{al} .^{10}$ reportan hallazgos similares; sin embargo, la leucocitosis sí se asoció con la severidad de la COVID-19 (OR: 3.46; IC 95\%: 1.0611.28; $p=0.04$ ). Al compararlo con los resultados obtenidos observamos que hay concordancia respecto a la linfopenia, aumento del INL y leucocitosis, no así con la disminución de $\mathrm{Hb}$ y plaquetas. Lo que pudiera deberse al hecho de que los estudios fueron realizados en población asiática y al inicio de la pandemia, además que lo que compararon fue la severidad del cuadro.

El INL ha sido propuesto como factor independiente de mortalidad, lo que se confirma con los resultados obtenidos, sin embargo, los resultados de la ROC muestran una baja exactitud para diferenciar entre mortalidad y necesidad de AVM. Aún no está claro el punto de corte del INL para predecir mortalidad. Liu, et al. ${ }^{11}$ sugieren que los pacientes mayores de 50 años con un INL $\geq 3.13$ desarrollarán una enfermedad críti$\mathrm{ca}$, mientras que Basbus, et al. ${ }^{12}$ sugieren un INL $>3$ para predecir una evolución desfavorable. Sin embargo, al utilizar dichos puntos de corte no se observa diferencia en relación con el desenlace.

Respecto a la hipercoagulabilidad sistémica, relacionada con el estado proinflamatorio severo, se ha reportado trombocitopenia entre el 58 y $95 \%$ de pacientes graves, asociándola a la mortalidad ${ }^{13}$, sin embargo, en algunos estudios, como el realizado por Lagadinou, et al. ${ }^{14}$ en Grecia, no se ha demostrado asociación 
significativa con la trombocitopenia. Esto concuerda con los resultados del presente estudio.

La presencia de anemia también ha sido propuesta como factor de mal pronóstico, por cómo puede afectar la oxigenación del organismo. Algassim, et al..$^{15}$ demostraron una correlación negativa entre la concentración de $\mathrm{Hb}$, la severidad de la enfermedad y la mortalidad. Sin embargo, al comparar las concentraciones de $\mathrm{Hb}$ no se observó diferencia significativa entre el grupo de pacientes que fallecieron y los que sobrevivieron, tampoco al clasificarlos en pacientes con y sin anemia, pudiendo deberse a la definición de anemia utilizada y las características propias de cada población.

A excepción de la trombocitopenia, coincidimos con Henry, et al. ${ }^{16}$, donde mediante un metaanálisis, comparando sobrevivientes y no sobrevivientes infectados por el SARS-CoV-2, no encontraron diferencia significativa en los niveles de $\mathrm{Hb}$, solo en la leucocitosis, la linfopenia y la trombocitopenia.

No obstante, Lippi, et al..$^{17}$ proponen la trombocitopenia como biomarcador de gravedad de la COVID-19, mostrando una OR de 5.1 (IC 95\%: 1.8-14.6) en un metaanálisis. Al comparar la media del recuento plaquetario y al clasificar como con o sin trombocitopenia en este estudio no se encontró diferencia significativa entre el grupo que falleció y el que sobrevivió, diferencia que puede corresponder al tamaño de muestra incluido.

El sexo masculino ha sido reportado con mayor probabilidad de muerte, sobre todo cuando tienen comorbilidades subyacentes ${ }^{18}$. Ruiz, et al. describen en población mexicana un predominio del sexo masculino $(60 \%)$ en los pacientes fallecidos ${ }^{19}$, coincidiendo con lo observado, donde el $58 \%$ de los pacientes fallecidos eran hombres, con una diferencia significativa en relación con el sexo; sin embargo, no fue factor de riesgo para mortalidad. Hay que tomar en cuenta que el estudio antes mencionado no hace una relación con la población que sobrevive, por lo que no pudieron hacer comparación entre grupos.

La edad y enfermedad cardiovascular preexistente es otro de los factores asociados a muerte por neumonía por SARS-CoV-20 ${ }^{20}$, coincidiendo con lo observado en que los pacientes mayores de 50 años mostraron mayor índice de defunción.

La escala SOFA ha sido utilizada como marcador pronóstico, se ha propuesto una puntuación mayor a 2 para predecir la mortalidad hospitalaria, con una sensibilidad del $83 \%$ y especificidad del $65.4 \%{ }^{21}$. Coincidiendo con lo observado, en donde considerando un punto de corte de 2 se confirma diferencia significativa en relación con la mortalidad.
Rodríguez, et al., en un estudio prospectivo en un tercer nivel de atención, sugieren como predictor de severidad la necesidad de ventilación mecánica invasiva de manera precoz ${ }^{22}$. Sin embargo, aunque sí hubo diferencia significativa en cuanto a los pacientes con y sin ventilación mecánica, un mayor porcentaje que no tuvo AVM fallecieron, probablemente por la gravedad con la que llegaron, no dando tiempo a intubarlos.

Es importante el tomar en cuenta las limitaciones del estudio, principalmente el diseño retrospectivo, monocéntrico, además del tamaño de muestra y datos con los que se disponía para ese momento, en el que a pesar de que se tomaron otros estudios que han sido propuestos como marcadores de mortalidad, como son la creatininfosfocinasa, la troponina, la mioglobina, la PCR y el dímero D, no todos los pacientes contaban con resultados, por lo que se optó por no incluirlos en el análisis.

No se cuenta con mucha información en el contexto de la población mexicana y es necesaria la realización de más estudios donde se relacionen los distintos biomarcadores tanto con la severidad como con la mortalidad y poder determinar de manera más confiable su uso, así como los puntos de corte de cada uno. Incluso poder confirmar o descartar si la trombocitopenia y la anemia no se relacionan con la mortalidad en pacientes con COVID-19, que ha sido fuente de controversia entre diversos estudios.

\section{Agradecimientos}

Al personal directivo por autorizarnos la revisión de expedientes y al personal de archivo y de laboratorio que nos facilitaron el acceso a los expedientes.

\section{Contribución de los autores}

Concepción y diseño del estudio: Patricia Seefoo Jarquin y Petrona Iveth Sánchez Sánchez. Adquisición de datos: Petrona Iveth Sánchez Sánchez, Dulce Cuatepotzo Xochihua. Análisis e interpretación de los datos: María de la Luz León Vázquez, Erik Castillo de la Peña. Borrador del artículo o la revisión crítica del contenido intelectual: María de la Luz León Vázquez, Patricia Seefoo Jarquin, Erik Castillo de la Peña, Petrona Iveth Sánchez Sánchez, Dulce Cuatepotzo Xochihua. Aprobación definitiva de la versión que se presenta: María de la Luz León Vázquez, Patricia Seefoo Jarquin, Erik Castillo de la Peña, Petrona Iveth Sánchez Sánchez, Dulce Cuatepotzo Xochihua. 


\section{Financiamiento}

La presente investigación no ha recibido ninguna beca específica de agencias de los sectores públicos, comercial o sin ánimo de lucro.

\section{Conflicto de intereses}

Los autores declaran no tener conflicto de intereses.

\section{Responsabilidades éticas}

Protección de personas y animales. Los autores declaran que para esta investigación no se han realizado experimentos en seres humanos ni en animales.

Confidencialidad de los datos. Los autores declaran que en este artículo no aparecen datos de pacientes.

Derecho a la privacidad y consentimiento informado. Los autores declaran que en este artículo no aparecen datos de pacientes.

\section{Bibliografía}

1. Guan WJ, Ni ZY, Hu Y, Liang WH, Ou CQ, He JX, et al. Clinical characteristics of coronavirus disease 2019 in China. N Engl J Med. 2020;382(18):1708-20.

2. Singh SP, Pritam M, Pandey B, Yadav TP. Microstructure, pathophysiology, and potential therapeutics of COVID-19: A comprehensive review. $J$ Med Virol. 2021;93(1):275-99.

3. Ponti G, Maccaferri M, Ruini C, Tomasi A, Ozben T. Biomarkers associated with COVID-19 disease progression. Crit Rev Clin Lab Sci. 2020;57(6):389-99.

4. Gao Y, Li T, Han M, Li X, Wu D, Xu Y, et al. Diagnostic utility of clinical laboratory data determinations for patients with the severe COVID-19. J Med Virol. 2020;92(7):791-6.

5. Singhal T. A review of coronavirus disease-2019 (COVID-19). Indian J Pediatr. 2020;87(4):281-6.

6. Du RH, Liang LR, Yang CQ, Wang W, Cao TZ, Li M, et al. Predictors of mortality for patients with COVID-19 pneumonia caused by SARS-CoV-2: a prospective cohort study. Eur Respir J. 2020;55(5):2000524.
7. Amgalan $A$, Othman $M$. Hemostatic laboratory derangements in COVID-19 with a focus on platelet count. Platelets. 2020;31(6):740-5.

8. Soraya GV, Ulhaq ZS. Crucial laboratory parameters in COVID-19 diagnosis and prognosis: An updated meta-analysis. Med Clin (Barc). 2020;155(4):143-51

9. Ghahramani S, Tabrizi R, Lankarani KB, Kashani SMA, Rezaei S, Zeidi N, et al. Laboratory features of severe vs. non-severe COVID-19 patients in Asian populations: a systematic review and meta-analysis. Eur J Med Res. 2020;25(1):30.

10. Li J, He X, Yuan Y, Zhang W, Li X, Zhang Y, et al. Meta-analysis investigating the relationship between clinical features, outcomes, and severity of severe acute respiratory syndrome coronavirus 2 (SARS-CoV-2) pneumonia. Am J Infect Control. 2021;49(1):82-9.

11. Liu J, Liu Y, Xiang P, Pu L, Xiong H, Li C, et al. Neutrophil-to-lymphocyte ratio predicts critical illness patients with 2019 coronavirus disease in the early stage. J Transl Med. 2020;18(1):206.

12. Basbus L, Lapidus MI, Martingano I, Puga MC, Pollán J. Índice neutrófilo-linfocito como factor pronóstico de COVID-19. Medicina (B Aires). 2020;80(Suppl 3):31-6.

13. Wool GD, Miller JL. The impact of COVID-19 disease on platelets and coagulation. Pathobiology. 2021;88(1):15-27

14. Lagadinou M, Salomou EE, Zareifopoulos N, Marangos M, Gogos C, Velissaris D. Prognosis of COVID-19: Changes in laboratory parameters. Infez Med. 2020;28(Suppl 1):89-95.

15. Algassim AA, Elghazaly AA, Alnahdi AS, Mohammed-Rahim OM, Alanazi AG, Aldhuwayhi NA, et al. Prognostic significance of hemoglobin level and autoimmune hemolytic anemia in SARS-CoV-2 infection. Ann Hematol. 2021;100(1):37-43.

16. Henry BM, de Oliveira MHS, Benoit S, Plebani M, Lippi G. Hematologic, biochemical and immune biomarker abnormalities associated with severe illness and mortality in coronavirus disease 2019 (covid-19): a meta-analysis. Clin Chem Lab Med. 2020:58(7):1021-8.

17. Lippi G, Plebani M, Henry BM. Thrombocytopenia is associated with severe coronavirus disease 2019 (COVID-19) infections: A meta-analysis. Clin Chim Acta. 2020:506:145-8.

18. Chen G, Wu D, Guo W, Cao Y, Huang D, Wang H, et al. Clinical and immunological features of severe and moderate coronavirus disease 2019. J Clin Invest. 2020;130:2620-9.

19. Ruiz-Quiñonez JA, Guzman-Priego CG, Nolasco-Rosales A, Tovilla-Zarate CA, Flores-Barrientos OI, Narváez-Osorio V, et al. Features of patients that died for COVID-19 in a hospital in the south of Mexico: A observational cohort study. PLoS One. 2021;16(2):e0245394.

20. Du RH, Liang LR, Yang CQ, Wang W, Cao TZ, Li M, et al. Predictor of mortality for patients with COVID-19 pneumonia caused by SARS-CoV-2: a prospective cohort study. Eur Respir J. 2020;55(5):2000524.

21. Lalueza A, Lora-Tamayo J, De la Calle C, Sayas-Catalán J, Arrieta E, Maestro G, et al. Utilidad de las escalas de sepsis para predecir el fallo respiratorio y la muerte en pacientes con COVID-19 fuera de las unidades de cuidados intensivos. Rev Clin Esp. Disponible online el 24 de Noviembre de 2020. DOI: https://doi.org/10.1016/j.rce.2020.10.004

22. Rodríguez A, Moreno G, Gómez J, Carbonell R, Picó-Plana E, Benavent Bofill C, et al. Severe infection due to the SARS-CoV-2 coronavirus: Experience of a tertiary hospital with COVID-19 patients during the 2020 pandemic. Med Intensiva. 2020;44(9):525-33. 